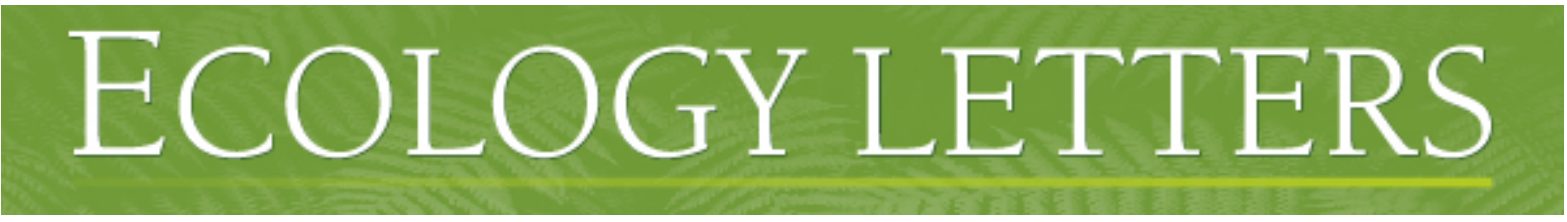

\title{
Unveiling ecological assembly rules from trait distributions
}

\begin{tabular}{|r|l|}
\hline Journal: & Ecology Letters \\
\hline Manuscript ID & ELE-01217-2020.R1 \\
\hline Manuscript Type: & Letters \\
\hline Author: & 14-Feb-2021 \\
\hline & $\begin{array}{l}\text { Complete List of Authors: } \\
\text { Singuet, Yoann } \\
\text { Linersity of Pierre; Czech Academy of Sciences, institute of botany; } \\
\text { Violle, Cyrille; CNRS, CEFE } \\
\text { Munoz, François; Université Grenoble Alpes, Laboratoire d'Ecologie } \\
\text { Alpine }\end{array}$ \\
\hline
\end{tabular}




\section{Letters}

2 Running head Trait diversity and assembly rules

3

4 Title: Unveiling ecological assembly rules from trait distributions

5

6 Nicolas Gross ${ }^{1, \dagger}, *$, Yoann Le Bagousse-Pinguet ${ }^{2, *}$, Pierre Liancourt ${ }^{3,4, *}$, Hugo Saiz ${ }^{5}$, Cyrille 7 Violle ${ }^{6}$, François Munoz ${ }^{7}$

8

$9{ }^{1}$ Université Clermont Auvergne, INRAE, VetAgro Sup, UMR Ecosystème Prairial, 63000 10 Clermont-Ferrand, France.

112 Aix Marseille Univ, CNRS, Avignon Université, IRD, IMBE, Technopôle Arbois-

12 Méditerranée Bât. Villemin - BP 80, F-13545 Aix-en-Provence cedex 04, France.

$13{ }^{3}$ Institute of Botany of the Czech Academy of Science, Průhonice, Czech Republic

$14{ }^{4}$ Plant Ecology Group, University of Tübingen, Tübingen, Germany

$15{ }^{5}$ Institute of Plant Sciences, University of Bern. Altenbergrain 21, 3013 Bern, Switzerland.

$16{ }^{6}$ Centre d'Écologie Fonctionnelle et Évolutive UMR 5175, Univ Montpellier - CNRS - EPHE

17 - IRD -Univ Paul Valéry Montpellier, 1919 route de Mende, 34293 Montpellier Cedex 5, 18 France

$19{ }^{7}$ Univ. Grenoble Alpes, LIPHY, F-38000 Grenoble, France

$21 \dagger$ Corresponding Author: nicolas.gross@inrae.fr

22

23 *these authors can be considered as co-first authors 


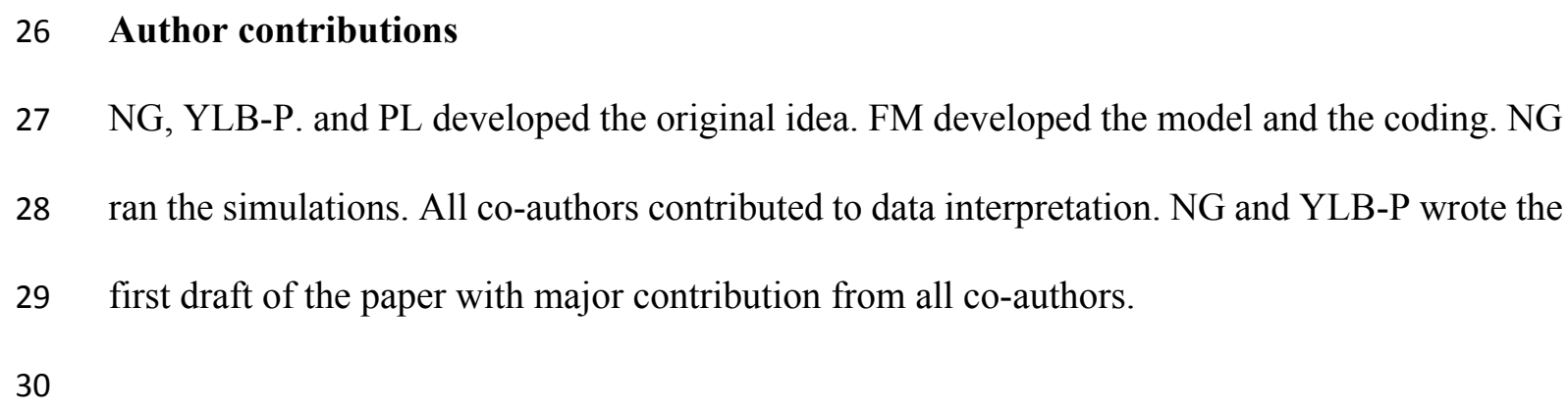


1

2

3

4

5

6

7

8

9 


\section{INTRODUCTION}

62 A basic tenet in community ecology is that trait diversity can reveal the influence of

63

64 deterministic processes on species assemblages (e.g., competition and abiotic factors; McGill et al. 2006; Weiher et al. 2011). Central to this research agenda is the hypothesis that high trait diversity reflects niche differentiation processes, while low trait diversity reflects the effect of environmental filtering selecting for species with similar trait values (Cornwell \& Ackerly 2009; Maire et al. 2012; Keddy 1992; Grime 2006). Much research has been devoted to analyze how patterns of trait diversity vary within and among communities (e.g., convergence or divergence) with the aim to uncover general assembly rules that could apply across many ecosystems (Diamond 1975; Weiher \& Keddy 2001). However, hypothesizing a direct and unequivocal linkage between patterns of trait diversity and assembly processes has proved too simplistic and fed much debate (Weiher et al. 2011; Götzenberger et al. 2012; Münkemüller et al. 2020).

Multiple assembly processes are likely to simultaneously influence local trait diversity, sometimes in opposite directions (Mayfield \& Levine 2010; Maire et al. 2012), making trait diversity patterns often difficult to distinguish from randomness (Götzenberger et al. 2012; Munoz \& Huneman 2016). The signature of deterministic processes can be blurred by stochastic processes such as demographic drift, or contingent variation in the regional species pool composition (Hubbell 2001; Spasojevic et al. 2018). Furthermore, most trait-based approaches have ignored the effect of species dispersal among communities (Spasojevic et al. 2014). Dispersal limitation can strongly reduce species richness (Ricklefs 1987), increasing the imprint of local demographic stochasticity (Leibold et al. 2004), and thereby yielding apparent randomness in community-level patterns (Götzenberger et al. 2012; Munoz \& Huneman 2016). Deciphering how multiple stochastic and deterministic processes shape trait diversity is not only crucial to expand our fundamental understanding of biodiversity patterns, but also to better predict the response of communities and ecosystems to ongoing environmental changes. 
Trait diversity is usually assessed using synthetic indices derived from the distribution of trait values within communities (e.g., Mason et al. 2005; Laliberté \& Legendre 2010; Enquist et al. 2015; Carmona et al. 2016). Most studies focus on the mean and variance/dispersion of trait distributions (Violle et al. 2012), or on related indices (e.g., FDis, Laliberté \& Legendre 2010). Considering that mean and variance suffice for describing trait diversity relies on the implicit assumption that trait values follow (approximately) a normal distribution. In essence, the normal distribution shows a bell shape, whose mean represents a local optimum that matches a given environment and the variance represents how trait values are constrained around this mean (Enquist et al. 2015, Fig. 1a). However, trait distributions often deviate from a normal distribution in real-world communities, and can exhibit asymmetric, flat, peaky or multimodal shapes (Fig. 1b) (Enquist et al. 2017; Le Bagousse-Pinguet et al. 2017). In such cases, further information on the shape of trait distributions is required to characterize trait diversity.

The shape of trait distributions can be quantified by the skewness and the kurtosis, which quantify the asymmetry and the evenness of the trait distributions, respectively. The skewness and the kurtosis are increasingly used in ecological research because they can provide insights on how species assemble within communities, and how they respond to ongoing environmental changes (Kraft et al. 2008; Enquist et al. 2015; Gross et al. 2017; Wieczynski et al. 2019). For instance, rapid environmental changes can simultaneously increase the skewness and kurtosis of trait distributions when a limited portion of the community with specific trait values benefit from the environmental change, and the recruitment of new species adapted to novel conditions is not immediate (see predictions in "Trait Driver Theory" in Enquist et al. 2015, 2017). Conversely, niche differentiation should decrease the kurtosis of trait distributions, yielding flatter or even bimodal distributions by promoting the coexistence of functionally contrasting species (Cornwell \& Ackerly 2009; Maire et al. 2012) . 
The skewness and the kurtosis are mathematically constrained through the Skewness-

113 Kurtosis Relationship (SKR hereafter), which can be used to characterize a broad spectrum of

114 distributions (Box 1) (Cullen \& Frey 1999). Across a variety of dryland plant communities,

115 Gross et al. (2017) reported that the distributions of plant height and specific leaf area followed

116 non-random SKRs. These empirical SKRs suggested the existence of general assembly rules

117 observable at the biome scale where traits organized according to specific families of trait

118 distributions (see Box 1 and Fig. 1c;d for a definition of family of trait distributions). Yet, we

119 do not know which ecological processes underpin such empirical assembly rules, how different

120 deterministic and stochastic processes modulate SKR patterns, and whether the signature of

121 these processes are distinguishable from one another. A theoretical evaluation of the SKR

122 framework is therefore needed to assess the effects of multiple stochastic and deterministic

123 assembly processes on trait distributions, and more generally, to advance our ability to identify

124 assembly rules from patterns of trait distributions.

125 In a theoretical experiment, we simulated the effects of multiple stochastic and 126 deterministic processes on trait diversity patterns. Using the ecolottery package in R language 127 (Munoz et al. 2018), we generated 800,000 communities spanning a broad spectrum of 128 ecological processes and of resulting trait distributions. Specifically, we considered four 129 community assembly scenarios: (i) a purely neutral scenario including stochastic processes 130 only; and (ii) three different "trait-based" scenarios, each combining stochastic processes with 131 a distinct outcome of deterministic processes entailing either convergence or divergence in trait 132 distributions (Loranger et al. 2018). Our goals were:

133 (1) to assess the extent to which the SKR and standard metrics of trait diversity capture 134 distinguishable signatures of assembly processes;

135 (2) to evaluate how the importance of stochastic processes (through the manipulation of 136 the regional species pool richness, and dispersal limitation among communities) and 
13

environmental variations across communities affect our ability to identify unambiguous signatures of the four community assembly scenarios.

\section{METHODS}

\section{Community assembly scenarios}

ecolottery is a modeling platform simulating the assembly of ecological communities (Munoz et al. 2018). It relies on a spatially implicit framework in which communities are assembled from an external pool of potential immigrants (e.g., a regional species pool) through dispersal. Stochastic and deterministic processes can be parameterized in ecolottery. They both influence the establishment success of immigrants and survival of their descendants in the model (Loranger et al. 2018; Munoz et al. 2018). Stochastic processes influence local diversity through demographic drift, dispersal limitation, and species richness in the regional pool (Etienne \& Alonso 2005). Deterministic processes modulate the success of immigrants based on how their trait values allow establishment and persistence in local environments.

We simulated three different outcomes of deterministic processes in ecolottery henceforth "trait-based filtering". The first trait-based filtering operates around a single optimal value defined by its matching with the local environmental conditions ("stabilizing filtering" hereafter), consistently with the classical view of the environmental filtering (Keddy 1992; Kraft et al. 2015). Stabilizing filtering typically generates normal or more leptokurtic distributions (e.g. hyperbolic distributions, Fig. 1 a,b), depending on the strength of the filtering (Enquist et al. 2015; Le Bagousse-Pinguet et al. 2017). The second trait-based filtering generates assemblages where alternative optimal trait values can confer greater species performance (“disruptive filtering"; Rolhauser \& Pucheta 2017; Loranger et al. 2018). This trait-based filtering produces patterns that reflect either niche differentiation among functionally contrasting species (Cornwell \& Ackerly 2009; Maire et al. 2012; Rolhauser \& 
162 Pucheta 2017). Disruptive filtering typically produces uniform or bimodal distributions (Fig.

163 1b). Finally, the third trait-based filtering generates assemblages in which species performance 164 varies monotonically with trait values ("Directional filtering"). This produces asymmetric trait 165 distributions (Loranger et al. 2018) (e.g. exponential distribution in Fig. 1b), e.g. in the case of 166 asymmetric light competition (Schamp et al. 2007) or directional environmental changes 167 (Enquist et al. 2015).

168

169

170

171

172

173

174

175

176

177

178

179

180

181

182

183

184

185

186

\section{In silico assembly experiment}

We considered four theoretical scenarios to assemble communities: (i) a purely neutral scenario that only considers the effect of stochastic processes on assembly without influence of trait differences on species performance and on trait distribution in assemblages (Hubbell 2001); and (ii) three contrasting scenarios in which deterministic processes (aforementioned traitbased filtering) are combined with stochastic processes. We modeled the four scenarios using different values of the parameter "filt" in ecolottery to represent the "neutral", "stabilizing", "disruptive" and "directional" scenarios (see R code in Appendix S1 in Supporting Information). In addition, we draw species trait values $i$ between -3 to +3 .

In a first simulation experiment, we simulated all communities with the same environmental conditions ("fixed environment", hereafter), under low or high dispersal limitation. Dispersal limitation was manipulated using a migration parameter " $m$ " that was set to either low $(m=0.95)$ or high dispersal limitation $(m=0.05)$. In a second simulation experiment, we simulated communities along an environmental gradient. The environmental gradient was simulated by adjusting the parameter "Env" from -2 to +2 , corresponding to the two extremes of a gradient (e.g., from cold to hot environments), and where 0 represented the mild environment (see Table S1 for model parameters). We chose the range of environmental variation "Env" (ranging from -2 to +2 ) to be lower than the range of possible trait values 
187 (ranging from -3 to +3 ) to avoid edge effects (Denelle et al. 2019). We set " $m$ " to either low or high dispersal limitation as explained in the first simulation experiment. The total number of individuals in the external species pool was set to 25,000 . This pool delineates the 'regional species pool' in our simulations. To evaluate how the richness of the species pool influenced community assembly, the pool of immigrants varied from 10 to 500 species. Richness at the regional level was fixed for each simulation run. The total number of individuals per community was set to 250 (Table S1), and we simulated 100 communities in each run. We chose these parameter values to approximate "realistic" vegetation sampling in the context of dryland (Gross et al. 2017, Table S1). Within communities, the number of individuals per species could vary according to the simulated assembly processes. The trait values of the regional species pool followed a uniform distribution, such that all trait values had equal probability of being selected. We randomly assigned trait values to each species. During this procedure, the model allowed different species to exhibit similar trait values, therefore allowing functional redundancy to occur among species (Munoz et al. 2018). For simplicity, we did not consider intraspecific trait variability in our simulations. Conspecific individuals thus displayed the same trait value.

\section{Analysis of simulated trait distributions}

We generated 800,000 communities: 500 runs where species richness varied from 10 to 500 species in the species pool $\times 100$ communities per run $\times 4$ scenarios $\times 2$ levels of dispersal limitation (low vs. high) $\times 2$ environmental contexts (a fixed environment vs. an environmental gradient). For each run, we recorded the total number of species in a given community (species richness). We quantified species relative abundance as the relative frequency of each species in each community (i.e., the number of individual of a species divided by the total number of individual in the community). 
212 We calculated the four moments of the trait distribution associated to each simulated 213 community: the mean, variance, skewness and kurtosis. Then, we calculated the parameters of 214 the SKRs across the 100 simulated distributions obtained from each run $\left(\mathrm{R}^{2}\right.$, slope, $\mathrm{Y}$-intercept 215 and the distance to the lower boundary; see Box 1). For each community, we also computed 216 classical indices of trait diversity using the dbFD function in the "FD" package in R [functional 217 dispersion (FDis), the Rao index, functional evenness (FEve); Laliberté and Legendre 2010]. 218 The four distribution moments and all trait diversity metrics were abundance-weighted. While 219 the SKR parameters were estimated across the 100 communities generated by each simulation 220 run, the four distribution moments, and taxonomic and trait diversity indices were calculated at 221 the community level, and thus generated 100 values for each run.

We evaluated whether the parameters of the SKRs, the four distribution moments and 223 all diversity metrics can be used to discriminate assembly processes from trait distributions. For 224 instance, lower variance, FDis and Rao values compared to the neutral scenario are expected 225 for the stabilizing and directional filtering, i.e., trait convergence. Conversely, higher variance, 226 FDis and Rao values compared to the neutral scenario are expected for the disruptive filtering, 227 i.e., trait divergence. To test for significant differences among scenarios, we used the "overlap" 228 package in R (Ridout \& Linkie 2009). For each diversity metric, we calculated the overlap 229 between each pair of scenarios and the mean overlap considering all scenarios together. Overlap 230 values ranged from 0 to 1 . An overlap $<0.05$ indicated that the metric under consideration 231 significantly discriminate assembly scenarios.

232

233 RESULTS

234 Discriminating scenarios using functional diversity metrics

235 The neutral scenario generated a wide range of trait diversity patterns, which challenges the 236 inference of ecological processes from trait distributions (Figs. 2, 3). Significant differences 
237 among scenarios were observed under low dispersal limitation. For instance, we observed

238 significant differences between the stabilizing and the neutral scenarios for FDis and for Rao

239 under low dispersal limitation (Figs. 2, 3). However, under high dispersal limitation, all trait

240 diversity indices - with the exception of the variance - simulated under the neutral scenario

241 systematically overlapped with those of the deterministic scenarios, meaning a lack of

242 differences among them.

243 The variance of trait distributions best discriminated assembly scenarios. We observed

244 a low mean overlap (Mo) when dispersal limitation was low and under fixed environment (Mo

$245=0.01$, Fig. 2a, left panel). The stabilizing and directional scenarios exhibited significantly

246 lower variances than those of the neutral scenario. However, the variance could not discriminate

247 the stabilizing from the disruptive and directional scenarios, and the neutral from the disruptive

248 scenario along an environmental gradient combined with high dispersal limitation $(\mathrm{Mo}=0.16$,

249 Fig. 2b). Overall, our results showed limited ability of these metrics of trait diversity to identify

250 unambiguous signatures of the four community assembly scenarios.

251

252 SKR parameters simulated in a fixed environment

253 Each studied scenario generated contrasting SKR patterns under fixed environmental 254 conditions and low dispersal limitation (Fig. 4, see also SKR plots in Fig. S1). The neutral and 255 stabilizing scenarios both generated weaker SKRs than the disruptive and directional scenarios 256 (significantly lower R ${ }^{2}$, Fig. 4b). These first two scenarios produced distributions that clustered 257 in the skewness-kurtosis space, and tended to overall converge toward a single skewness258 kurtosis coordinate as species richness in the regional pool increased (from dark to bright blue 259 dots, Fig. 4a). In contrast, both the directional and the disruptive scenarios yielded strong SKRs, 260 i.e. characterized by significantly higher $\mathrm{R}^{2}$ than neutral and stabilizing scenarios (Fig. 4b). 261 Specifically, the disruptive scenario generated SKRs with the highest $\mathrm{R}^{2}$, ranging from 0.6 to 
2620.99 (Fig. 4b), indicating that all communities assembled under this scenario belong to a unique

263 family of trait distributions (skew-bimodal distribution, Fig. 1c, d).

264 The SKRs generated from the four scenarios had also clear and significantly distinct Y-

265 intercepts (Fig. 4b). Therefore, the SKR not only discriminated the three scenarios containing

266 deterministic processes from a purely neutral scenario, but also clearly differentiated these three

267 scenarios from one another. The neutral scenario had a Y-intercept $=1.86$ (Fig. 4b) suggesting

268 that it converged toward uniform distribution (Fig. 1b). This pattern of trait diversity observed

269 at the Y-intercept of the SKR thus mirrored the trait distribution of the regional pool. The

270 stabilizing scenario produced communities that converged toward a normal distribution (Y-

271 intercept $=2.82$ Fig. $4 \mathrm{~b}$ ). This scenario generated a peakier and therefore less even trait

272 distribution than the neutral one. The directional scenario generated distributions that were

273 mostly asymmetric, and that converged toward a skewness value $\sim 1$ as species richness

274 increased (Fig. 4a) and a Y-intercept $=2.1$ (Fig. 4b). The disruptive scenario had the lowest Y-

275 intercept $=1.33$ (Fig. 4b). This scenario generated a family of trait distributions approaching

276 the lower boundary of the skewness-kurtosis space, corresponding to highly platykurtic

277 bimodal distributions (see the skew-bimodal distribution in Fig. 1c). Therefore, the disruptive

278 scenario was the one with the highest trait diversity in our simulations.

279 Increasing the influence of stochasticity, through high dispersal limitation, reduced the

280 species richness at the community level (Fig. S2). It consistently increased the asymmetry of

281 trait distributions for all scenarios, thereby increasing the scatter of distributions in the

282 skewness-kurtosis space (Fig. 4a). Higher scattering of skewness and kurtosis values produced

283 SKRs with higher $\mathrm{R}^{2}$ for all scenarios (Fig. 4b). For instance, the $\mathrm{R}^{2}$ of the neutral scenario

284 ranged from 0.25 to 0.75 (Fig. 4b), indicating that significant SKRs can be observed and

285 produced by a high stochasticity even under a strictly neutral scenario. Yet, irrespective of the

286 degree of stochasticity, the disruptive filtering always produced the strongest SKRs (Fig. 4b). 
Furthermore, increasing dispersal limitation marginally influenced the Y-intercept of the SKRs.

The mean overlap among scenarios remained extremely low under high dispersal limitation (Yintercept: Mo $=0.03$, Fig. 4b). We observed significant differences between the stabilizing, neutral and disruptive scenarios, although the directional scenario overlapped with the neutral one (overlap $=0.18)$. These results suggested that the SKR approach is therefore able to differentiate a neutral assembly scenario from the effect of environmental filtering (stabilizing scenario) and niche differentiation (disruptive scenario) even under a high stochasticity.

SKR parameters simulated along an environmental gradient

Changing environmental conditions across communities affected local mean trait value for all the studied scenarios except the neutral one (Fig. S3). Furthermore, the variance was constant

\section{DISCUSSION}

\section{Commonalities in trait distributions across multiple communities unveil assembly rules}

311 view that "simple stochastic models can reproduce natural diversity patterns" (Hubbell 2005). 
312 Likewise, deterministic assembly scenarios generated a wide range of trait distributions

313 (Enquist et al. 2015; Le Bagousse-Pinguet et al. 2017). The ambiguity among scenarios further

314 increased in our simulations as stochasticity increased or when the environmental conditions

315 changed (e.g., sampling performed along environmental gradients). Therefore, our study

316 therefore demonstrates that distinguishing deterministic from purely neutral patterns based on

317 standard trait-based metrics, or by the four moments of trait distributions taken separately, is

318 particularly challenging.

319 The SKR framework provided far less ambiguous insights into underlying assembly

320 processes, as contrasting assembly scenarios exhibited distinct SKR patterns. The SKR

321 approach did not only well discriminate the neutral from trait-based filtering scenarios, but also

322 clearly differentiated each of the filtering scenarios from one another, irrespective of the degree

323 of dispersal limitation, environmental condition or the size of the species pool. The SKR

324 approach appears therefore as a promising tool to unveil assembly processes from trait

325 distributions, even under high stochasticity, a yet important limitation of existing approaches

326 aiming at interpreting trait diversity patterns of real-world communities (Weiher et al. 2011;

327 Münkemüller et al. 2020).

328 The SKR framework shifts our perspective to focus on commonalities in trait 329 distributions - rather than differences - to unveil assembly rules. For instance, the disruptive 330 filtering generated a set of symmetric and asymmetric distributions. Under this scenario, 331 increasing environmental constraints simultaneously reduced the variance and increased both 332 the skewness and the kurtosis of trait distributions (Enquist et al. 2015; Le Bagousse-Pinguet 333 et al. 2017), consistently with the environmental filtering hypothesis (Keddy 1992). However, 334 the trait distributions all aligned along a specific SKR. The observed SKR pattern thus described 335 a family of trait distributions, in which each single distribution represents an instance of a more 336 general trait distribution operating at larger spatial scale (e.g., at the biome scale in Gross et al. 
337 2017). All distributions within this family shared a common property: trait distributions are 338 bimodal (Fig. S5), thus reflecting the effect of niche differentiation processes promoting the coexistence of functionally contrasting species (Maire et al. 2012). In other words, even if different environmental conditions along a gradient (and stochastic processes) can affect the shape parameters of distributions by increasing the skewness and the kurtosis separately, a bimodality persists as the signature of niche differentiation. By characterizing commonalities

343 in trait distributions, the SKR represents a critical step toward the identification of general rules 344 governing the functional organization of species assemblages

By identifying families of trait distributions, the SKR framework scales up trait distributions from the community to broader geographical scales (e.g. landscape / regional / continental / biome scales). The SKR framework differs from other trait-based approaches aiming at addressing biogeographical questions by aggregating trait patterns across spatial scales (Violle et al. 2014; Carmona et al. 2016). While the SKR framework scales up trait distributions from local to wider geographical scales, it also keeps the information on the shape of trait distributions observed at the community scale. In other words, the SKR framework increases the spatial extent by quantifying trait distributions at large spatial scale but does not reduce the grain size. Therefore, the SKR framework represents an opportunity to merge community ecology and biogeography and to "achieve a deeper understanding of biodiversity and its change across communities" (Chase et al. 2018).

The skewness and the kurtosis of trait distributions are increasingly used to detect the short term effects of global change on communities, and may be used as a signal of compositional changes (Enquist et al. 2017; Griffin-Nolan et al. 2019; Wieczynski et al. 2019;

359 Zhang et al. 2019; Aguirre-Gutiérrez et al. 2020). Yet, variations in skewness and kurtosis are 360 often considered independently, i.e. without accounting for their mathematical dependency 361 (Box 1). Observing a decrease in trait evenness (higher kurtosis) may entirely rely on changes 
362 in the degree of skewness. Accounting for the SKR when assessing the impact of global change

363 on ecological communities should provide additional insights on how the ecological reassembly

364 under environmental change, and may provide a more robust validation of theoretical

365 predictions (e.g. the "Trait Driver Theory": Enquist et al. 2015, 2017). Focusing on the distance

366 to the lower boundary of the SKR (see Box 1) (Liu et al. 2020) or the deviation of individual

367 communities from empirical to random SKR (Gross et al. 2017) - rather than raw variations in

368 kurtosis - may help diagnosing shifts in community evenness and community disassembly

369 under ongoing environmental change.

370

371 Linking theoretical predictions to empirical patterns of trait diversity

372 Our simulations suggest that niche differentiation and dispersal limitation jointly shape the trait

373 diversity in real-world communities. Combining the disruptive scenario with high dispersal

374 limitation was the only case that generated a strong family of trait distributions (i.e., a SKR

375 with a $\mathrm{R}^{2}>0.90$ ). This family of trait distributions exhibited lower kurtosis than under the

376 neutral scenario, a pattern similar to the empirical SKRs observed in drylands worldwide (Gross

377 et al. 2017). When simulating along an environmental gradient, the disruptive scenario also

378 reproduced the impact of environmental filtering by reducing variance and increasing the

379 kurtosis within communities (Fig. S3), and the shift in dominance of contrasting functional

380 groups (e.g. the shrub-to-grass shift in dominance commonly observed in drylands; Fig. S4,

381 Bestelmeyer et al. 2018). Altogether, our results corroborate the view that dryland communities

382 are shaped by a combination of these multiple drivers.

383 The modelling platform ecolottery provides a simple and flexible environment to

384 simulate the effect of multiple stochastic and deterministic processes on trait distributions

385 (Munoz et al. 2018). Further implementations are needed to provide reliable and quantitative 
386

387

388

389

390

391

392

393

394

395

396

397

398

399

400

401

402

403

404

405

406

407

408

409

410

predictions when comparing theoretical predictions with observed trait distributions in realworld communities:

First, different assembly processes have been shown to act differently on traits describing contrasted axes of functional specialization (e.g. size vs. resource use related-traits, Kraft et al. 2008; Cornwell \& Ackerly 2009; Maire et al. 2012). On the one hand, the disruptive scenario may well apply to resource-use traits as contrasting strategies for resources acquisition and utilization may favor species coexistence (Maire et al. 2012). On the other hand, the directional scenario could constitute a valuable hypothesis for size-related traits in light limited environments where asymmetric competition predominates (Schamp et al. 2007; Gross et al. 2009). Considering the effect of multiple assembly processes acting on independent functional dimensions would allow a more realistic representation of community assembly and species coexistence (Maire et al. 2012).

Second, the distributions of trait values at large spatial scale (e.g. at the scale of the regional species pool) are important drivers determining local assembly processes and trait diversity (Carmona et al. 2016; Le Provost et al. 2017; Spasojevic et al. 2018). To evaluate how regional trait pools influence SKR patterns, we conducted a sensitivity analysis using different trait distributions at the regional scale (see Fig. S5 for details). Our results show that the regional trait pool influenced the SKR parameters for each scenario. It supports the need to account for the regional trait pool to provide reliable predictions on trait diversity within and across communities (Carmona et al. 2016; Spasojevic et al. 2018). Nevertheless, we also observed that the relative differences among contrasting assembly scenarios remained consistent despite variation in the composition of the regional trait pool, highlighting the robustness of the SKR approach.

Third, downscaling from a global / regional trait pool to the local community scale may require the development of a spatially-explicit framework, as contrasting assembly processes 
411 may act simultaneously but at different spatial scale (Keddy 1992; Spasojevic et al. 2014; Le

412 Provost et al. 2017). Such framework would allow evaluating how environmental filtering

413 acting at broad spatial scale (Kraft et al. 2015; Le Bagousse-Pinguet et al. 2017) interacts with

414 local biotic processes (Maire et al. 2012), e.g. by simulating the stabilizing and disruptive

415 scenarios within a single framework but acting at contrasted spatial scale. In this context, further

416 implementations of the model would allow for a more explicit representation of biotic

417 interactions. For instance, the disruptive scenario considers a selection of two optima due to

418 alternative suitable strategies (e.g., coexisting guilds, Cornwell \& Ackerly 2009; Maire et al.

419 2012). However, the disruptive scenario does not account for interactions among individual

420 species trough density-dependent mechanisms (MacArthur \& Levins 1967) as well as for other

421 types of biotic interactions (e.g., facilitation and indirect interactions) that have been shown to

422 impact trait diversity in plant communities (Gross et al. 2009; Saiz et al. 2019).

423

424 Conclusions

425 Here we show that the skewness-kurtosis relationship (SKR) offers a powerful mean to evaluate 426 the effect of multiple stochastic and deterministic processes on the assembly of ecological 427 communities. By focusing on the co-variation between the skewness and the kurtosis across 428 multiple communities, the SKR framework identifies commonalities in the shape of the trait 429 distributions that can serve as a basis to infer assembly processes. The SKR framework is robust 430 to stochastic processes such as variation in regional species and trait pools, dispersal limitation. 431 Finally, our simulations suggest that the joint effect of local-scale processes such as niche 432 differentiation and regional-scale dispersal limitation can have key implications for shaping 433 biodiversity. Our study offers promising avenue for identifying ecological assembly rules in 434 real-world ecosystems. 


\section{Acknowledgments}

437 We thank the editor Brian Enquist and the three anonymous reviewers whose 438 comments/suggestions helped improve and clarify this manuscript; Miguel Berdugo, Lucas 439 Deschamps, Fernando T. Maestre, Pascal Monestiez and Caroline Tucker for fruitful 440 discussions. YLB-P was supported by a Marie Sklodowska-Curie Actions Individual 441 Fellowship (MSCA-IF) within the European Program Horizon 2020 (DRYFUN Project 442 656035). PL received funding from the Czech Science Foundation (GACR 17-19376S) and the 443 Czech Academy of Sciences (RVO 67985939). CV was supported by the European Research 444 Council (ERC) Starting Grant Project 'ecophysiological and biophysical constraints on 445 domestication in crop plants' (grant ERC-StG-2014-639706-CONSTRAINTS). This study was 446 partly supported by the French Foundation for Research on Biodiversity (FRB; $447<$ www.fondationbiodiversite.fr $>$ ) and EDF in the context of the CESAB project 'causes and 448 consequences of functional rarity from local to global scales' (FREE). N.G. was supported by 449 the AgreenSkills + fellowship programme which has received funding from the EU's Seventh 450 Framework Programme under grant agreement N FP7-609398 (AgreenSkills + contract).

451

452

\section{REFERENCES}

Aguirre-Gutiérrez, J., Malhi, Y., Lewis, S.L., Fauset, S., Adu-Bredu, S., Affum-Baffoe, K., et al. (2020). Long-term droughts may drive drier tropical forests towards increased functional, taxonomic and phylogenetic homogeneity. Nature communications, 11, 110.

Bestelmeyer, B.T., Peters, D.P.C., Archer, S.R., Browning, D.M., Okin, G.S., Schooley, R.L., et al. (2018). The Grassland-Shrubland Regime Shift in the Southwestern United States: Misconceptions and Their Implications for Management. BioScience, 68, 678690. 
461 Carmona, C.P., de Bello, F., Mason, N.W.H. \& Lepš, J. (2016). Traits Without Borders:

462

463

464

465

466

467

468

469

470

471

472

473

474

475

476

477

478

479

480

481

482

483

484

485 Integrating Functional Diversity Across Scales. Trends in Ecology \& Evolution, 31, $382-394$.

Chase, J.M., McGill, B.J., McGlinn, D.J., May, F., Blowes, S.A., Xiao, X., et al. (2018). Embracing scale-dependence to achieve a deeper understanding of biodiversity and its change across communities. Ecology Letters, 21, 1737-1751.

Cornwell, W.K. \& Ackerly, D.D. (2009). Community assembly and shifts in plant trait distributions across an environmental gradient in coastal California. Ecological Monographs, 79, 109-126.

Cristelli, M., Zaccaria, A. \& Pietronero, L. (2012). Universal relation between skewness and kurtosis in complex dynamics. Phys. Rev. E, 85, 066108.

Cullen, A.C., Frey, H.C. \& Frey, C.H. (1999). Probabilistic techniques in exposure assessment: a handbook for dealing with variability and uncertainty in models and inputs. Springer Science \& Business Media.

Denelle, P., Violle, C. \& Munoz, F. (2019). Distinguishing the signatures of local environmental filtering and regional trait range limits in the study of trait-environment relationships. Oikos, 128, 960-971.

Diamond, J.M. (1975). The island dilemma: lessons of modern biogeographic studies for the design of natural reserves. Biological conservation, 7, 129-146.

Enquist, B.J., Bentley, L.P., Shenkin, A., Maitner, B., Savage, V., Michaletz, S., et al. (2017). Assessing trait-based scaling theory in tropical forests spanning a broad temperature gradient. Global Ecology and Biogeography, 26, 1357-1373.

Enquist, B.J., Norberg, J., Bonser, S.P., Violle, C., Webb, C.T., Henderson, A., et al. (2015). Chapter Nine - Scaling from Traits to Ecosystems: Developing a General Trait Driver Theory via Integrating Trait-Based and Metabolic Scaling Theories. In: Advances in 
Ecological Research, Trait-Based Ecology - From Structure to Function (eds. Pawar, S., Woodward, G. \& Dell, A.I.). Academic Press, pp. 249-318.

Etienne, R.S. \& Alonso, D. (2005). A dispersal-limited sampling theory for species and alleles: A dispersal-limited sampling theory. Ecology Letters, 8, 1147-1156.

Götzenberger, L., de Bello, F., Brlaathen, K.A., Davison, J., Dubuis, A., Guisan, A., et al. (2012). Ecological assembly rules in plant communities—approaches, patterns and prospects. Biological reviews, 87, 111-127.

Griffin-Nolan, R.J., Blumenthal, D.M., Collins, S.L., Farkas, T.E., Hoffman, A.M., Mueller, K.E., et al. (2019). Shifts in plant functional composition following long-term drought in grasslands. Journal of Ecology, 107, 2133-2148.

Grime, J.P. (2006). Trait convergence and trait divergence in herbaceous plant communities: mechanisms and consequences. Journal of Vegetation Science, 17, 255-260.

Gross, N., Kunstler, G., Liancourt, P., Bello, F.D., Suding, K.N. \& Lavorel, S. (2009). Linking individual response to biotic interactions with community structure: a traitbased framework. Functional Ecology, 23, 1167-1178.

Gross, N., Le Bagousse-Pinguet, Y., Liancourt, P., Berdugo, M., Gotelli, N.J. \& Maestre, F.T. (2017). Functional trait diversity maximizes ecosystem multifunctionality. Nature ecology \& evolution, 1, 0132.

Hubbell, S.P. (2001). The Unified Neutral Theory of Biodiversity and Biogeography (MPB32). Princeton University Press.

Hubbell, S.P. (2005). Neutral theory in community ecology and the hypothesis of functional equivalence. Functional ecology, 19, 166-172.

Keddy, P.A. (1992). Assembly and response rules: two goals for predictive community ecology. Journal of vegetation science, 3, 157-164. 
510 Kraft, N.J., Adler, P.B., Godoy, O., James, E.C., Fuller, S. \& Levine, J.M. (2015).

511 Community assembly, coexistence and the environmental filtering metaphor.

$512 \quad$ Functional ecology, 29, 592-599.

513 Kraft, N.J.B., Valencia, R. \& Ackerly, D.D. (2008). Functional Traits and Niche-Based Tree

514 Community Assembly in an Amazonian Forest. Science, 322, 580-582.

515 Laliberté, E. \& Legendre, P. (2010). A distance-based framework for measuring functional

516 diversity from multiple traits. Ecology, 91, 299-305.

517 Le Bagousse-Pinguet, Y., Gross, N., Maestre, F.T., Maire, V., de Bello, F., Fonseca, C.R., et

518 al. (2017). Testing the environmental filtering concept in global drylands. Journal of

$519 \quad$ Ecology, 105, 1058-1069.

520 Le Provost, G., Gross, N., Börger, L., Deraison, H., Roncoroni, M. \& Badenhausser, I. (2017).

521 Trait-matching and mass effect determine the functional response of herbivore

522 communities to land-use intensification. Funct Ecol, 31, 1600-1611.

523 Leibold, M.A., Holyoak, M., Mouquet, N., Amarasekare, P., Chase, J.M., Hoopes, M.F., et al.

524 (2004). The metacommunity concept: a framework for multi-scale community

525 ecology: The metacommunity concept. Ecology Letters, 7, 601-613.

526 Liu, C., Li, Y., Zhang, J., Baird, A.S. \& He, N. (2020). Optimal Community Assembly

527 Related to Leaf Economic-Hydraulic-Anatomical Traits. Frontiers in plant science,

$528 \quad 11,341$.

529 Loranger, J., Munoz, F., Shipley, B. \& Violle, C. (2018). What makes trait-abundance

530 relationships when both environmental filtering and stochastic neutral dynamics are at

$531 \quad$ play? Oikos, 127, 1735-1745.

532 MacArthur, R. \& Levins, R. (1967). The limiting similarity, convergence, and divergence of

533 coexisting species. The american naturalist, 101, 377-385. 
534 Maire, V., Gross, N., Börger, L., Proulx, R., Wirth, C., Pontes, L. da S., et al. (2012). Habitat

535 filtering and niche differentiation jointly explain species relative abundance within 536

537

538 grassland communities along fertility and disturbance gradients. New Phytologist, 196, 497-509.

Mason, N.W.H., Mouillot, D., Lee, W.G. \& Wilson, J.B. (2005). Functional richness, functional evenness and functional divergence: the primary components of functional diversity. Oikos, 111, 112-118.

Mayfield, M.M. \& Levine, J.M. (2010). Opposing effects of competitive exclusion on the phylogenetic structure of communities. Ecology letters, 13, 1085-1093.

McGill, B.J., Enquist, B.J., Weiher, E. \& Westoby, M. (2006). Rebuilding community ecology from functional traits. Trends in ecology \& evolution, 21, 178-185.

Münkemüller, T., Gallien, L., Pollock, L.J., Barros, C., Carboni, M., Chalmandrier, L., et al. (2020). Dos and don'ts when inferring assembly rules from diversity patterns. Global Ecology and Biogeography.

Munoz, F., Grenié, M., Denelle, P., Taudière, A., Laroche, F., Tucker, C., et al. (2018). ecolottery: Simulating and assessing community assembly with environmental filtering and neutral dynamics in R. Methods in Ecology and Evolution, 9, 693-703.

Munoz, F. \& Huneman, P. (2016). From the Neutral Theory to a Comprehensive and Multiscale Theory of Ecological Equivalence. The Quarterly Review of Biology, 91, $321-342$.

Ricklefs, R.E. (1987). Community diversity: relative roles of local and regional processes. Science, 235, 167-171.

Ridout, M.S. \& Linkie, M. (2009). Estimating overlap of daily activity patterns from camera trap data. JABES, 14, 322-337. 
558 Rolhauser, A.G. \& Pucheta, E. (2017). Directional, stabilizing, and disruptive trait selection 559 as alternative mechanisms for plant community assembly. Ecology, 98, 668-677.

560 Saiz, H., Le Bagousse-Pinguet, Y., Gross, N. \& Maestre, F.T. (2019). Intransitivity increases 561 plant functional diversity by limiting dominance in drylands worldwide. Journal of $562 \quad$ Ecology, 107, 240-252.

563 Schamp, B.S., Chau, J. \& Aarssen, L.W. (2007). Dispersion of traits related to competitive $564 \quad$ ability in an old-field plant community. J Ecology, 0, 071119203335008-???

565 Shmida, A. \& Ellner, S. (1984). Coexistence of plant species with similar niches. Vegetatio, $566 \quad 58,29-55$.

567 Spasojevic, M.J., Catano, C.P., LaManna, J.A. \& Myers, J.A. (2018). Integrating species traits $568 \quad$ into species pools. Ecology, 99, 1265-1276.

569 Spasojevic, M.J., Copeland, S. \& Suding, K.N. (2014). Using functional diversity patterns to 570 explore metacommunity dynamics: a framework for understanding local and regional 571 influences on community structure. Ecography, 37, 939-949.

572 Violle, C., Enquist, B.J., McGill, B.J., Jiang, L.I.N., Albert, C.H., Hulshof, C., et al. (2012).

573 The return of the variance: intraspecific variability in community ecology. Trends in $574 \quad$ ecology \& evolution, 27, 244-252.

575 Violle, C., Reich, P.B., Pacala, S.W., Enquist, B.J. \& Kattge, J. (2014). The emergence and $576 \quad$ promise of functional biogeography. $P N A S, 111,13690-13696$.

577 Weiher, E., Freund, D., Bunton, T., Stefanski, A., Lee, T. \& Bentivenga, S. (2011). Advances, 578 challenges and a developing synthesis of ecological community assembly theory. 579 Philosophical Transactions of the Royal Society B: Biological Sciences, 366, 2403$580 \quad 2413$

581 Weiher, E. \& Keddy, P. (2001). Ecological assembly rules: perspectives, advances, retreats. $582 \quad$ Cambridge University Press. 
583 Wieczynski, D.J., Boyle, B., Buzzard, V., Duran, S.M., Henderson, A.N., Hulshof, C.M., et 584 al. (2019). Climate shapes and shifts functional biodiversity in forests worldwide. 585 Proceedings of the National Academy of Sciences, 116, 587-592.

586 Zhang, D., Peng, Y., Li, F., Yang, G., Wang, J., Yu, J., et al. (2019). Trait identity and 587 functional diversity co-drive response of ecosystem productivity to nitrogen 588 enrichment. Journal of Ecology, 107, 2402-2414.

\section{SUPPORTING INFORMATION}

591 Additional Supporting Information may be downloaded via the online version of this article at 592 Wiley Online Library (www.ecologyletters.com).

593 As a service to our authors and readers, this journal provides supporting information supplied 594 by the authors. Such materials are peer-reviewed and may be re-organized for online delivery, 595 but are not copy-edited or typeset. Technical support issues arising from supporting information 596 (other than missing files) should be addressed to the authors. 
598 BOX 1: The Skewness-Kurtosis Relationship (SKR)

599 We examined whether the relationship between the skewness and the kurtosis of trait 600 distributions (Fig. 1a) can help deciphering the signatures of contrasting assembly processes.

601 The approach is inspired by optimization procedures increasingly used in physics, climatology 602 and economy (e.g. Cristelli et al. 2012). We apply the approach to diagnose assembly rules 603 from trait distributions (the Skewness-Kurtosis Relationship [SKR] approach). While the mean 604 and variance reflect the location and the scale of a distribution (the latter being the dispersion 605 of trait values within a community), the skewness and kurtosis inform on its shape. The degree 606 of skewness quantifies the asymmetry of a given distribution. For instance, a skew distribution 607 indicates the dominance of extreme trait values (see exponential distribution in Fig. 1b), which 608 can typically arise from asymmetric competition for light (Schamp et al. 2007). The kurtosis 609 quantifies the relative peakiness of a trait distribution and the relative density of its tails. Low 610 kurtosis values reflect an even distribution of trait values within a given community, a definition 611 of a high trait diversity (Gross et al. 2017). Low Kurtosis may reflect the coexistence of 612 functionally contrasting species (see uniform and bimodal distributions in Fig. 1b) (Enquist et 613 al. 2017; Gross et al. 2017). In contrast, peaked distributions characterized by high kurtosis 614 value reflect a low trait diversity, and may typically occur under strong environmental filtering 615 (sensu Keddy 1992) selecting for a limited range of trait value (see hyperbolic distribution in 616 Fig. 1b).

617 Skewness (S) and kurtosis (K) are related through the following inequality (Fig. 1b):

$618 \quad \mathrm{~K} \geq \beta \mathrm{S}^{\wedge} 2+\alpha$

619 This inequality generates a mathematically constrained triangle in which all possible trait 620 distributions can be represented and characterized (Gross et al. 2017), i.e. the skewness-kurtosis 621 space. For instance, the normal distribution is defined by a unique combination of skewness 622 and kurtosis values of 0 and 3 respectively. It can therefore be represented as a single coordinate 
623 in the skewness-kurtosis space (red dot, in Fig. 1d). Families of trait distributions can also be 624 represented by a skewness-kurtosis relationship (SKR, hereafter) with a slope $\beta$ and a Y625 intercept $\alpha$. A SKR implies that when trait distributions become more skewed, they also become 626 more peaked, resulting in a decrease in evenness. The slope $\beta$ of the SKR measures the strength 627 of the relationship, i.e., the extent to which evenness decreases as trait distributions become 628 more skewed. The Y-intercept $\alpha$ indicates the lowest kurtosis value at skewness $=0$, and 629 corresponds to the highest trait diversity predicted by a given SKR. Distributions belonging to 630 a family of distributions share common properties. For instance, in the case of a skew-bimodal 631 distribution (Fig. 1c, green dashed line in Fig. 1d), all distributions are bimodal although their 632 degree of skewness and kurtosis can vary across communities. This would be the case when 633 two distinct functional groups coexist within communities (e.g. grass and shrub species) but their relative abundance can vary across communities.

The inequality (1) has a lower boundary that sets a limit to the minimal kurtosis value predicted for any degree of skewness, i.e. the potential maximum trait diversity for a given skewness (black dash line, $\mathrm{K}=\mathrm{S}^{2}+1$, Fig. 1b) (see Gross et al. 2017 for a mathematical demonstration). The distance to the lower boundary for a given distribution - exemplified with the black arrow in the case of the exponential distribution (Fig. 1b) - thus quantifies the extent to which trait diversity departs from the potential maximum trait diversity independently from the degree of skewness. Although skewness and kurtosis individually provide valuable information on community trait distributions, the SKR approach helps to diagnose complex trait distributions (Cullen \& Frey 1999) and to reveal the extent to which trait diversity is allow identifying assembly rules through the identification of commonalities in the shape of the trait distributions observed across multiple communities. maximized within communities. Applying the SKR framework to ecological communities may 


\section{Figures Legend:}

649 Figure 1 Characterizing complex trait distributions using the Skewness-Kurtosis Relationship 650 (SKR). (a) A trait distribution is a density function representing the relative frequency or 651 abundance of trait values within a community. Examples of trait distributions include normal 652 (panel a), uniform, bimodal, hyperbolic or exponential distributions (panel b). (c) Example of 653 a family of distributions: a skew-bimodal distribution (see results, and Box 1). (d) The 654 Skewness-Kurtosis space. Trait distributions are characterized by distinct skewness (S) 655 kurtosis $(\mathrm{K})$ coordinates. Families of trait distributions can be characterized by a specific 656 Skewness-Kurtosis Relationship (SKR) such as $\mathrm{K}=\beta \mathrm{S}^{2}+\alpha$. The distance to the lower boundary 657 (i.e. potential minimum kurtosis value) of a given distribution (exemplified with the black arrow 658 in the case of the exponential distribution) quantifies the extent to which trait evenness is 659 maximized.

660

661 Figure 2 The four moments of the trait distributions simulated under the four theoretical 662 scenarios (Neutral [Neu], Stabilizing [Sta], Disruptive [Dis], Directional [Dir]) under (a) fixed 663 environment and varying species pools, (b) changing environment and fixed species pool, and 664 under low / high dispersal limitation. We represent the mean, variance, skewness and kurtosis 665 of the trait distributions simulated under each scenario using violin plots. For each panel, we 666 provide the mean overlap (Mo) among the four moments of the trait distributions. Different 667 letters indicate significant differences between scenarios (overlap $<0.05$; NS for Not668 Significant).

669

670 Figure 3 Species richness (Sp. Rich.) and commonly-used trait diversity indices (FDis, Rao,

671 FEve) simulated under the four theoretical scenarios (Neutral [Neu], Stabilizing [Sta], 672 Disruptive [Dis], Directional [Dir]) in (a) Fixed environment varying species pools, (b) 
673 changing environment fixed species pool, and under low / high dispersal limitation. We

674 represent each predicted parameter using violin plots. For each panel, we provide the mean

675 overlap (Mo) between the four-parameter distributions. Different letters indicate significant

676 differences between scenarios (overlap < 0.05; but NS for Not-Significant).

677

678 Figure 4 Effect of the different assembly scenarios on the Skewness-Kurtosis Relationship 679 (SKR) in a fixed environment and varying regional species pool. (a) Coordinates of the 680 simulated communities in the skewness-kurtosis space under the different scenarios. We 681 simulate 100 communities (number of run $=500$ ) assembled in a constant environment under 682 low / high dispersal limitation. Dark and light blue dots represent poor and rich regional pools,

683 respectively. (b) We represent the parameters of the SKRs ( $\mathrm{R}^{2}$, Y-intercept (alpha), slope of the 684 SKRs (beta), distance to the lower boundary) for each scenario using violin plots. We provide 685 the mean overlap (Mo) among the four scenarios. Different letters indicate significant 686 differences between scenarios (overlap < 0.05; but NS for Not-Significant).

687

688 Figure 5 Effect of the different assembly scenarios on the Skewness-Kurtosis Relationship 689 (SKR) along an environmental gradient. (a) Coordinates of the simulated communities in the 690 skewness-kurtosis space under the different scenarios. We simulated 100 communities (number 691 of run $=500$ ) assembled along an environmental gradient (from Env $=-2$ to Env $=+2$ ) and a 692 fixed regional species pool $(\mathrm{n}=150$ species $)$ under low / high dispersal limitation. Dark and 693 light blue dots represent the environmental gradient ranging from -2 to +2 . (b) We represent 694 the parameters of the SKRs ( $\mathrm{R}^{2}$, Y-intercept (alpha), slope of the SKRs (beta), distance to the 695 lower boundary) for each scenario using violin plots. We provide the mean overlap (Mo) among 696 the four scenarios. Different letters indicate significant differences between scenarios (overlap $697<0.05$; but NS for Not-Significant). 


\section{FIGURES}

(a) Trait distribution

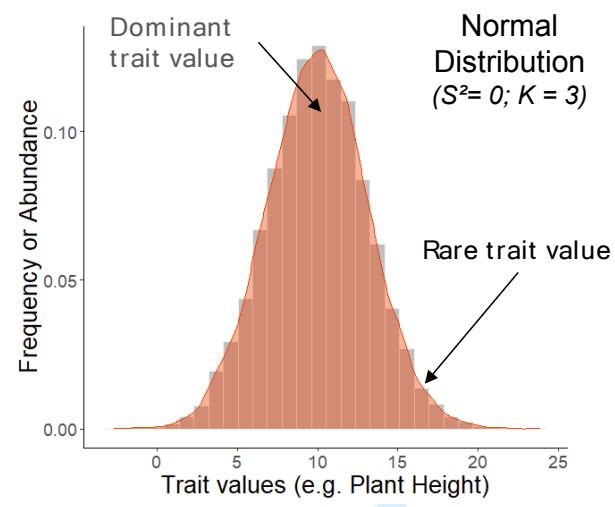

(b) Example of trait distributions
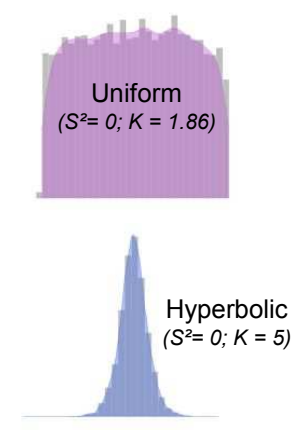

699

700

701 (c) Example of a family of trait distributions

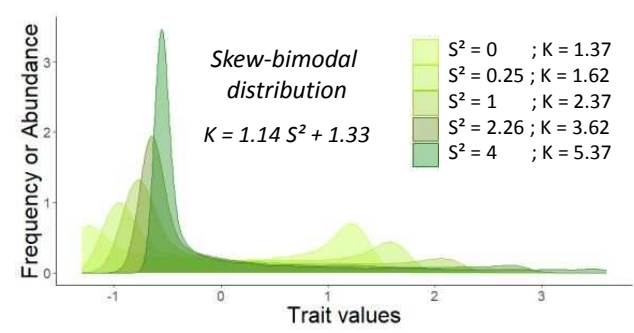

(d) The Skewness-Kurtosis Relationship $\left(K=\alpha+\beta S^{2}\right)$

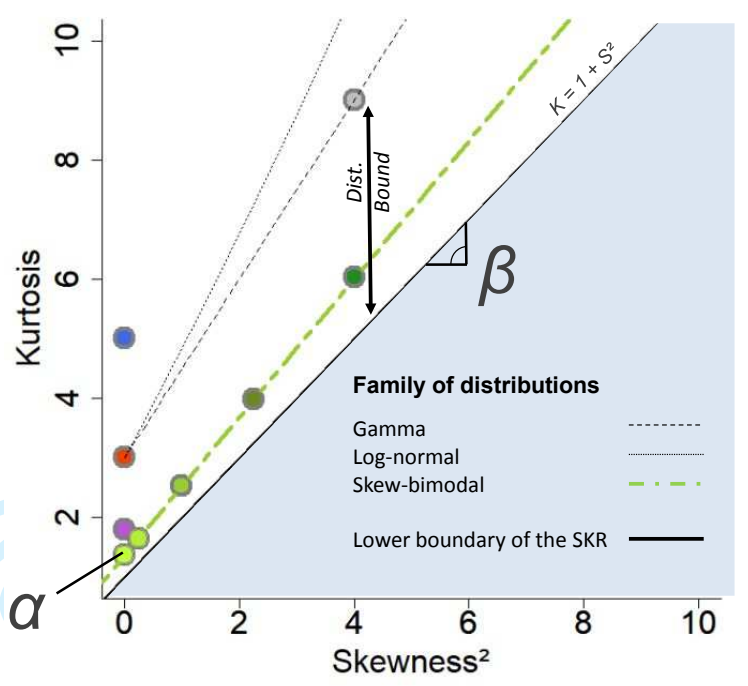


(a) Fixed Environment \& Varying Species Pool
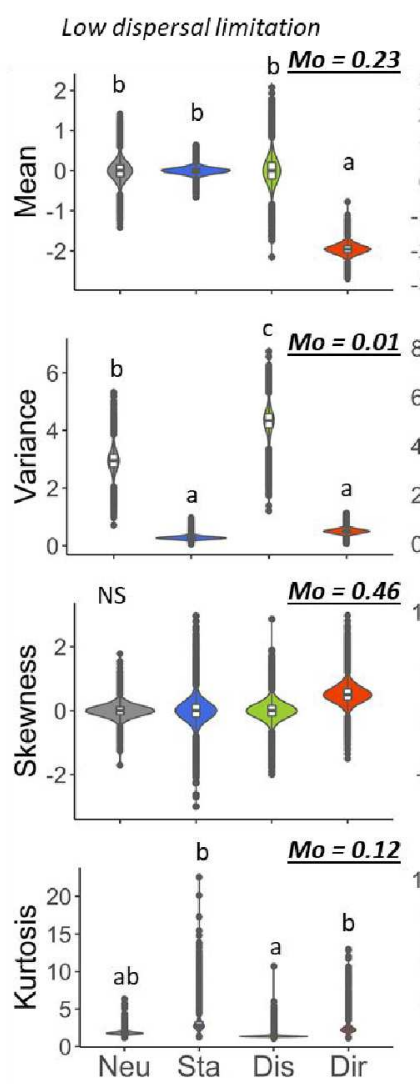

High dispersal limitation
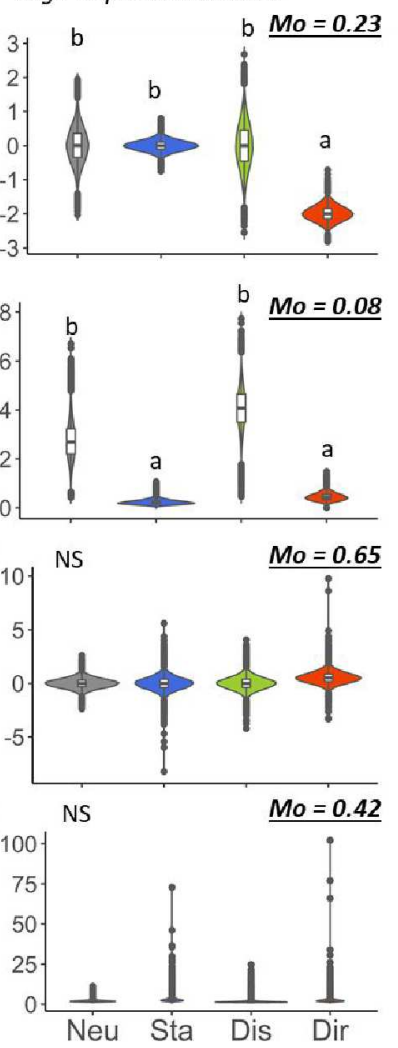

(b) Environmental gradient \& Fixed Species Pool

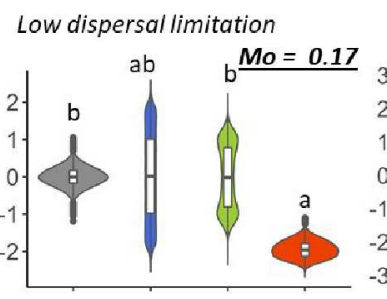

High dispersal limitation
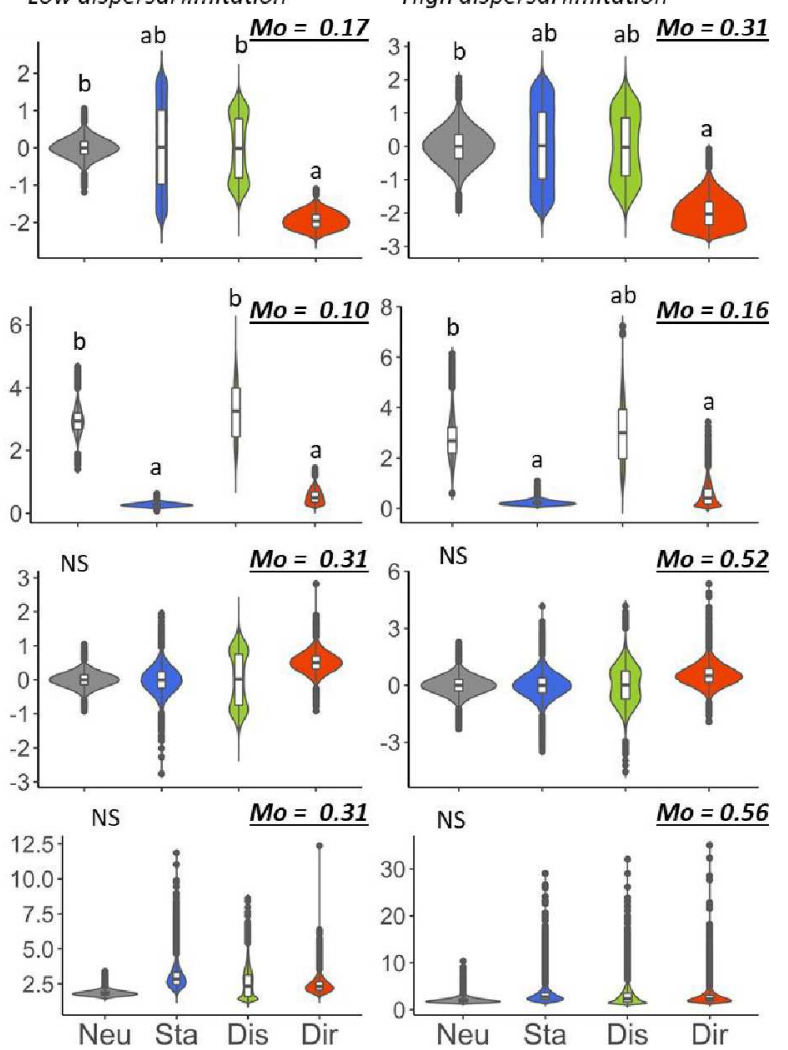

702

703

Figure 2

704 
Ecology Letters

Page 32 of 34

(a) Fixed Environment \& Varying Species Pool
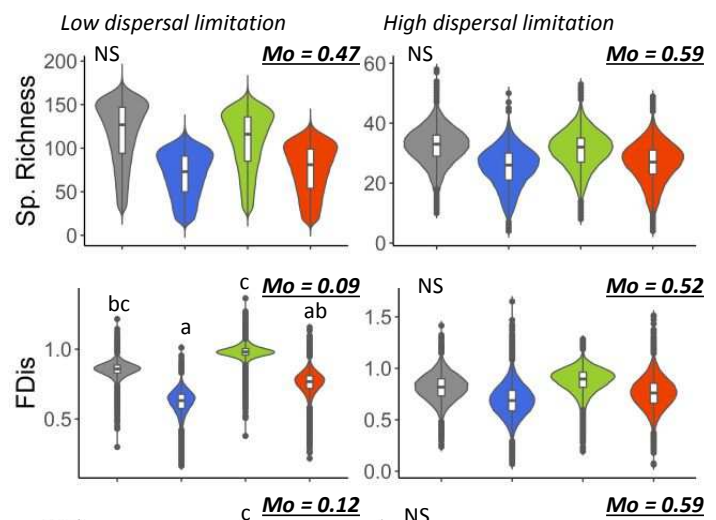

19

20

21

22

23

24

25

26

27

28

29

30

31

32

33

34

35

36

37

38

39

40

41

42

43

44

45

46

47

48

49

50

51

52

53

54

55

56

57

58

59

60

Figure 3

708
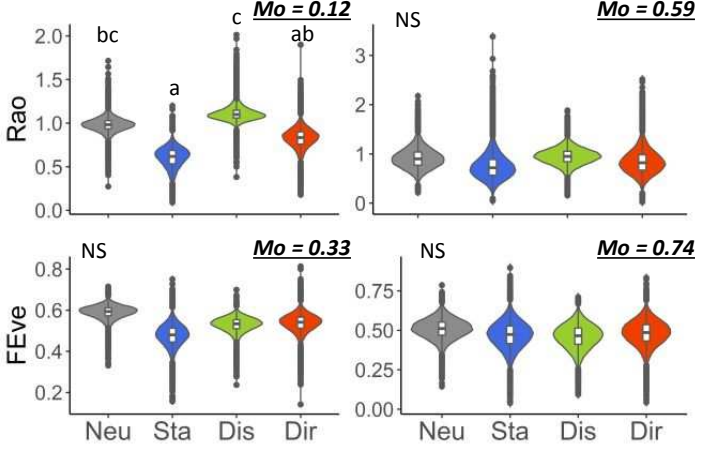

(b) Environmental gradient \& Fixed Species Pool
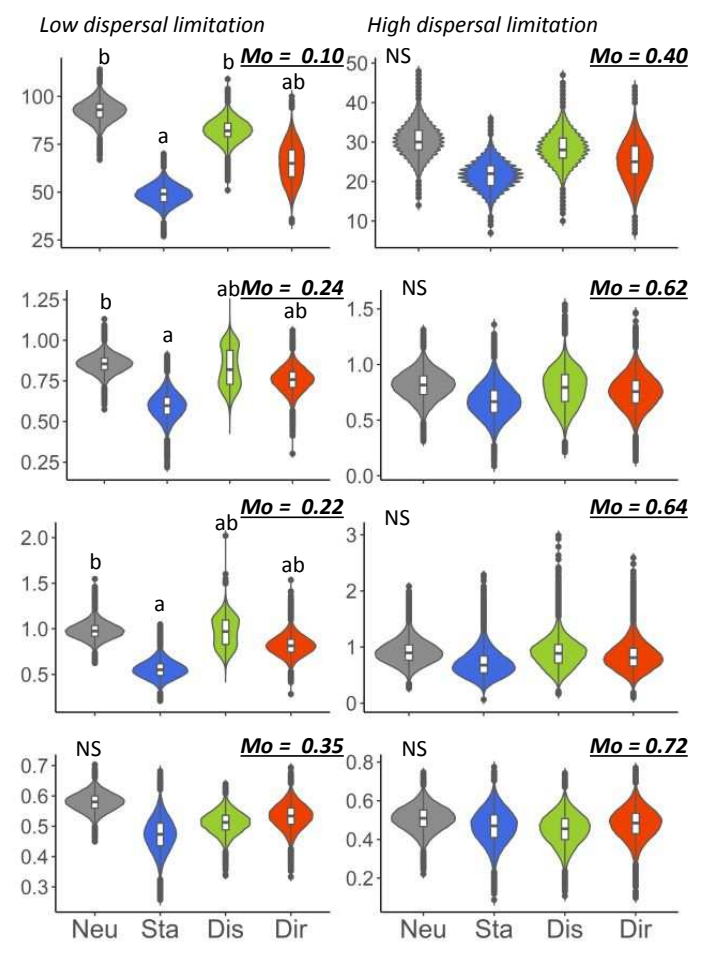

32 


\section{(a) Skewness-Kurtosis space}

Neutral scenario (Neu)
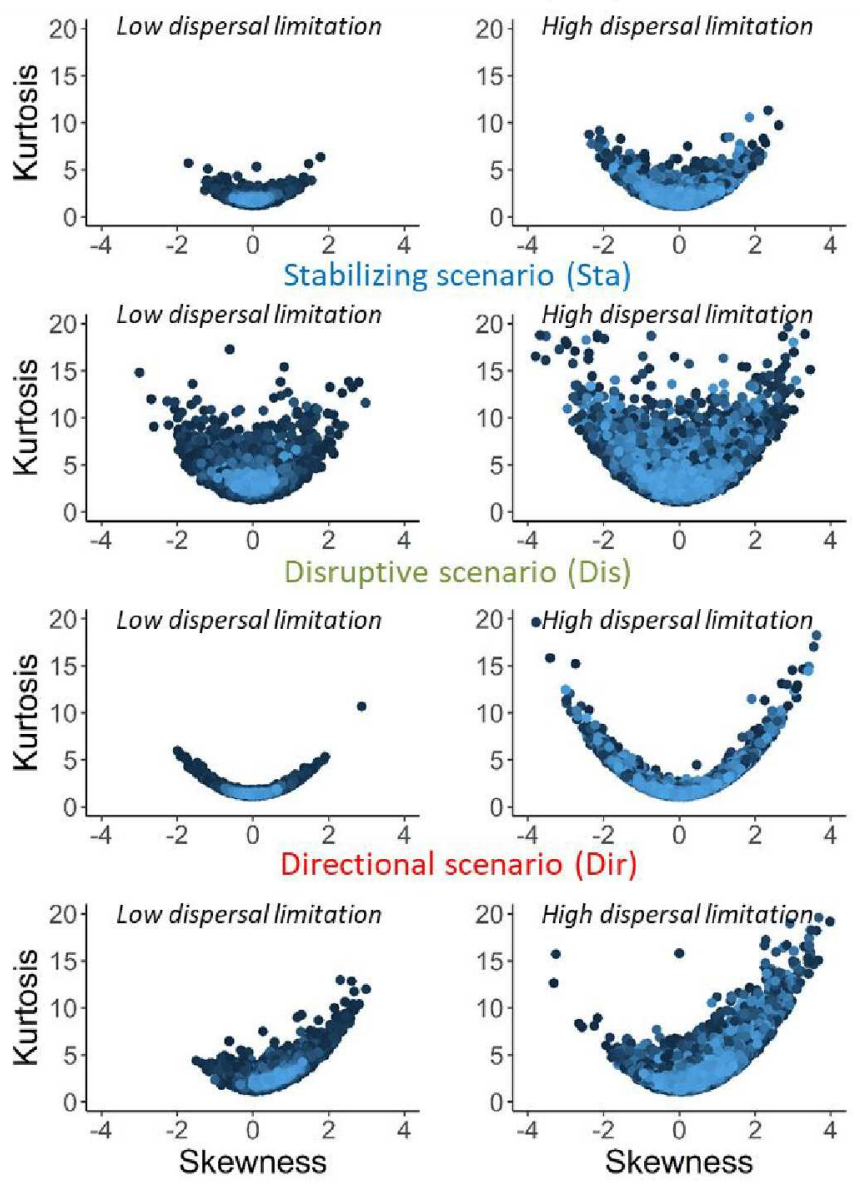

709

710

\section{Figure 4}

(b) SKR parameters

Low dispersal limitation
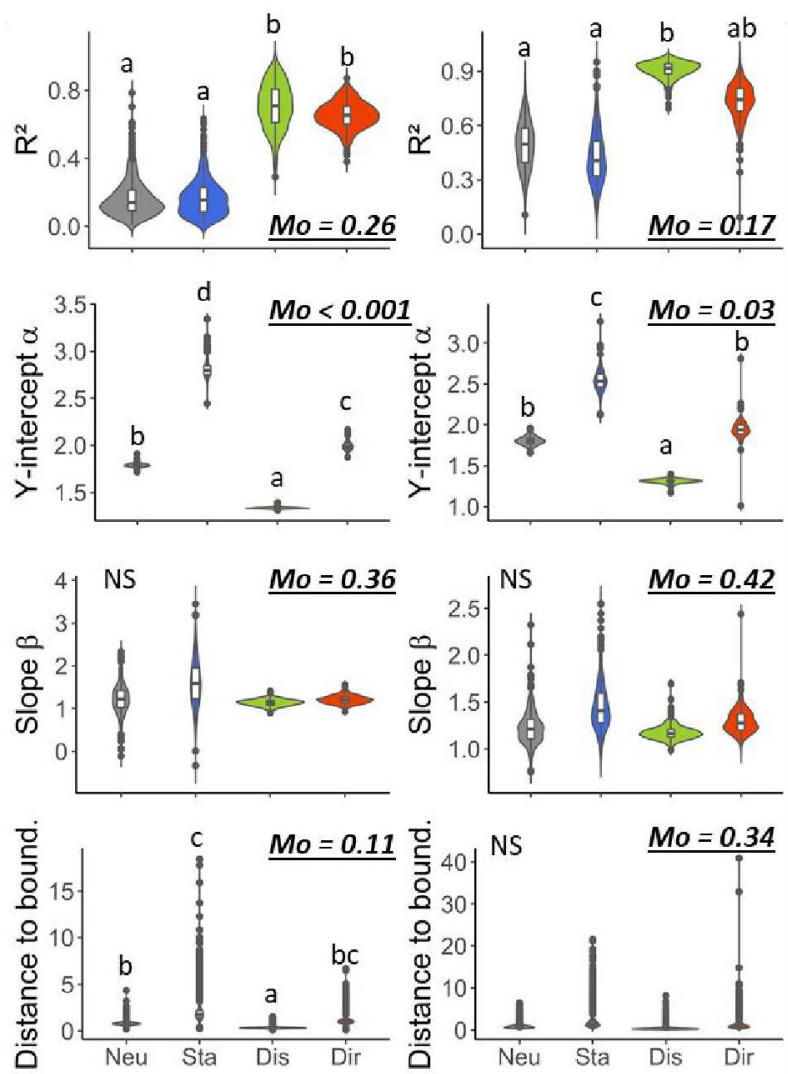

$M o=0.34$

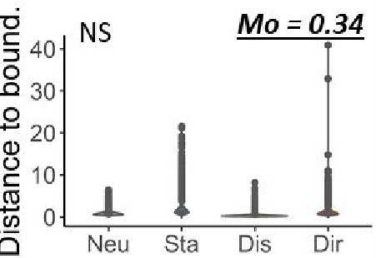




\section{(a) Skewness-Kurtosis space}

Neutral scenario (Neu)
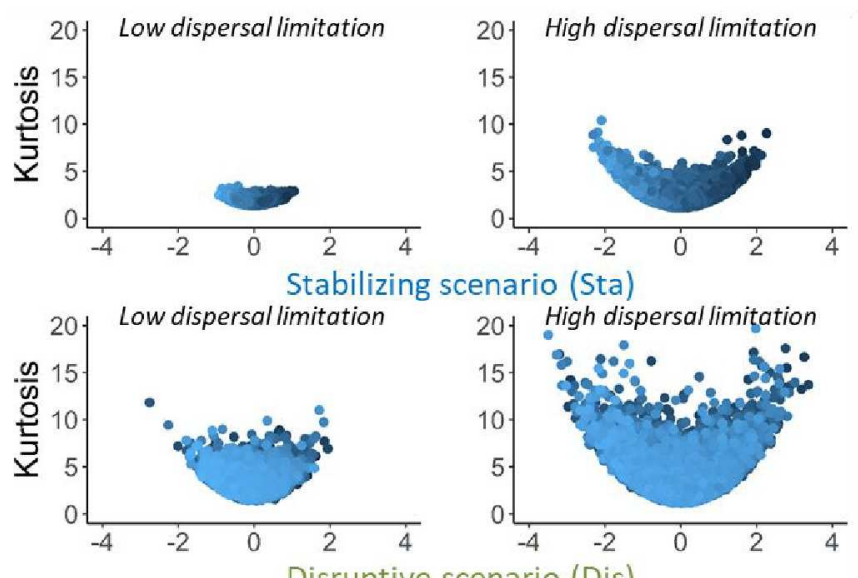

Disruptive scenario (Dis)

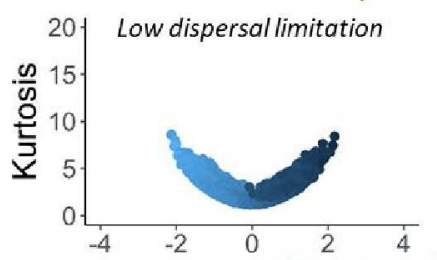

$$
\text { Directional }
$$
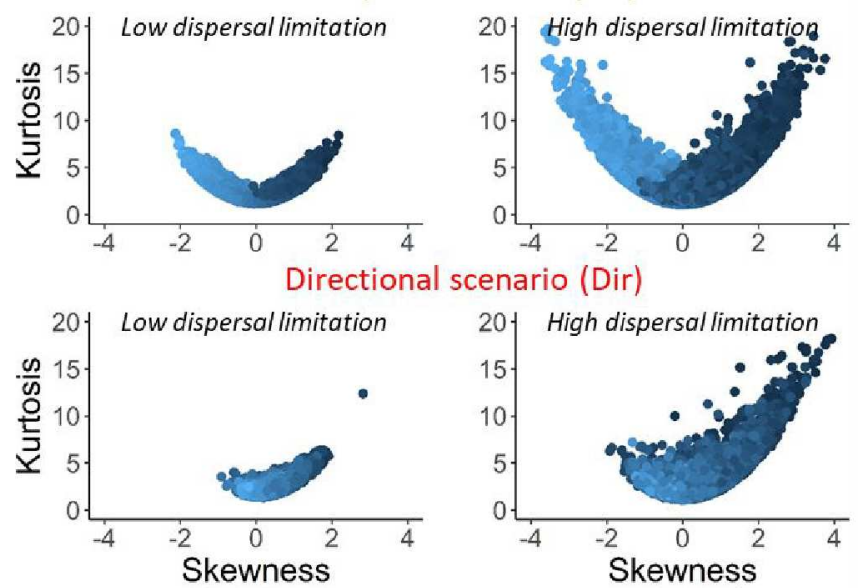

713

714

715

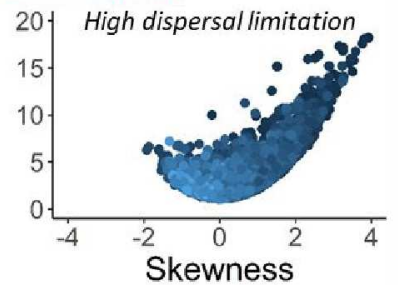

Figure 5

\section{(b) SKR parameters}

No dispersal limitation

Dispersal limitation
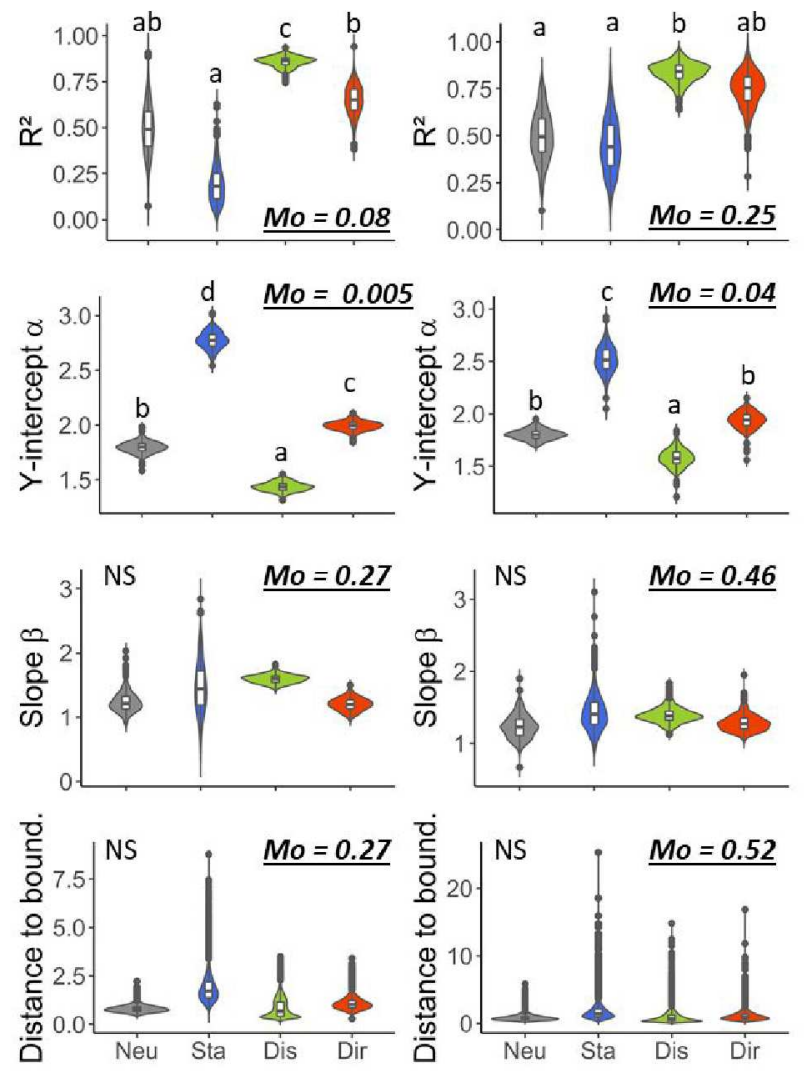

36

37

38

39

40

41

42

43

44

45

46

47

48

49

50

51

52

53

54

55

56

57

58 